\title{
Disminución de la fracción de expulsión del ventrículo izquierdo por estrés con adenosina como predictor de isquemia
}

\author{
Gabriela Meléndez Ramírez, ${ }^{1}$ Edith Liliana Posada-Martínez, ${ }^{1}$ Aloha Meave-González, ${ }^{1}$ \\ Rafael Vera-Urquiza ${ }^{1}$ y Juan Betuel Ivey-Miranda ${ }^{2}$ \\ 'Secretaría de Salud, Instituto Nacional de Cardiología Ignacio Chávez, Departamento de Resonancia Magnética Cardiovascular; ${ }^{2}$ Instituto Mexicano \\ del Seguro Social, Centro Médico Nacional Siglo XXI, Hospital de Cardiología. Ciudad de México, México
}

\section{Resumen}

Introducción: En estudios de medicina nuclear se ha observado que la disminución de la fracción de expulsión del ventrículo izquierdo (FEVI) se asocia con enfermedad coronaria trivascular; en resonancia magnética cardiaca (RMC) no se ha estudiado el papel que desempeña la disminución de la FEVI. Objetivo: Evaluar la asociación entre la disminución de la FEVI y la isquemia cardiaca en pacientes con estudios de RMC con adenosina. Método: Estudio transversal comparativo. Los criterios de inclusión fueron pacientes evaluados con RMC con adenosina entre enero de 2009 y junio de 2015. Se comparó el cambio en la FEVI en pacientes con estudio positivo versus pacientes con estudio negativo para isquemia por este método. Resultados: Se incluyeron 59 pacientes: 41 del sexo masculino (70\%), edad de $59.7 \pm 10.9$ años; 38 de los estudios fueron positivos para isquemia. La delta de la FEVI (FEVI postestrés - FEVI reposo) fue de $-0.16 \pm 5.9$ versus $5.3 \pm 4.7$ $(p<0.001)$ en pacientes con y sin isquemia, respectivamente. Conclusión: Los pacientes con estudios positivos para isquemia tuvieron menor delta FEVI que los que tuvieron estudios negativos para isquemia.

PALABRAS CLAVE: Resonancia magnética cardiaca. Enfermedad arterial coronaria. Fracción de expulsión del ventrículo izquierdo.

\begin{abstract}
Background: The decrease in left ventricular ejection fraction (LVEF) has been observed to be associated with three-vessel coronary disease in nuclear medicine studies; however, the role played by LVEF decrease has not been studied with cardiovascular magnetic resonance (CMR). Objective: To assess the association between LVEF decrease and cardiac ischemia in patients with CMR studies with adenosine. Method: Cross-sectional, comparative study. Inclusion criteria were: patients assessed with CMR with adenosine between January 2009 and June 2015. LVEF change was compared between patients testing positive for ischemia versus those who tested negative. Results: Fifty nine patients were included: 41 were males (70\%), mean age was $59.7 \pm 10.9$ years; $38 \%$ of the studies tested positive for ischemia. Delta LVEF (post-stress LVEF resting LVEF) was $-0.16 \pm 5.9$ versus $5.3 \pm 4.7(p<0.001)$ in patients with and without ischemia, respectively. Conclusion: Patients who tested positive for ischemia had lower delta LVEF than those with negative studies for ischemia.
\end{abstract}

KEY WORDS: Cardiovascular magnetic resonance. Coronary artery disease. Left ventricular ejection fraction.

\section{Correspondencia:}

Edith Liliana Posada-Martínez

E-mail: liliposada1701@gmail.com
Fecha de recepción: 22-02-2017

Fecha de aceptación: 06-02-2018

DOI://dx.doi.org/10.24875/GMM.18003322
Gac Med Mex. 2018;154:315-319

Disponible en PubMed www.gacetamedicademexico.com 


\section{Introducción}

La enfermedad arterial coronaria (EAC) causa aproximadamente $42 \%$ de todas las muertes relacionadas con enfermedades cardiovasculares. ${ }^{1}$ En México, las enfermedades del corazón son la principal causa de muerte. ${ }^{2}$ La angiografía coronaria invasiva es el estándar de oro en el diagnóstico de la enfermedad arterial coronaria, pero no se considera el estudio inicial, de tal forma que el diagnóstico puede ser apoyado por pruebas funcionales (prueba de esfuerzo 0 prueba de imagen con estrés). ${ }^{3,4}$ La gammagrafía es un recurso probado para la evaluación de la perfusión miocárdica; ${ }^{4,5}$ permite valorar simultáneamente la perfusión ventricular izquierda, función y volúmenes. ${ }^{5}$ Las imágenes de perfusión sincronizadas con el electrocardiograma o gated-SPECT, posestrés y en reposo, ha permitido valorar el fenómeno de aturdimiento posisquémico; la magnitud de la depresión de la fracción de expulsión del ventrículo izquierdo (FEVI) posestrés en relación con el reposo correlaciona con la severidad de la isquemia. Bestetti et al. ${ }^{4,5}$ reportaron que la reducción significativa de la FEVI posestrés en gated-SPECT se debe a incremento del volumen telesistólico (VTS) causado por aturdimiento endocárdico. En la actualidad se sabe que la dilatación isquémica transitoria y la disminución de la FEVI postestrés son importantes predictores de enfermedad arterial coronaria grave en estudios con SPECT. ${ }^{6}$

La resonancia magnética cardiaca (RMC) es un método cada vez más utilizado debido a su alta resolución, planos de imagen ilimitados y capacidad de proveer diferentes tipos de información en un solo estudio; es considerada el estándar de oro para la cuantificación volumétrica y funcional. ${ }^{7-9}$ La cuantificación de la perfusión con RMC demostró buena correlación con medidas de reserva de flujo fraccional, estándar de oro para el diagnóstico de isquemia miocárdica. ${ }^{8}$ En cuanto a la cardiopatía isquémica, la RMC con estrés puede ser utilizada para detectar anormalidades del movimiento de la pared inducidas por isquemia; el análisis visual permite observar áreas de perfusión reducida y con asistencia computacional identificar el realce durante la fase de primer paso; 9,10 su rendimiento diagnóstico es comparable al otros métodos. ${ }^{11-13}$

A pesar de las utilidades de la RMC con adenosina, no existe información contundente acerca de la comparación de parámetros funcionales en el postestrés respecto del reposo, como en otras técnicas de imagen, por ejemplo, la falta de incremento o disminución de la FEVI posterior al estrés (delta FEVI $\leq 0 \%$ ) asociada con mayor probabilidad de EAC grave en SPECT.

\section{Método}

Estudio transversal comparativo con muestreo no aleatorio de pacientes consecutivos a quienes se les realizó RMC con adenosina por sospecha o conocimiento de EAC, entre enero de 2009 y junio de 2015. Se recolectó información demográfica y clínica de los expedientes de los pacientes. Se consideró que el paciente tenía "EAC conocida" si tenía el antecedente de coronariografía y angioplastia.

En este estudio, el estándar de referencia para el diagnóstico de cardiopatía isquémica fue que la RMC mostrara resultados inequívocos de isquemia miocárdica.

La RMC se realizó con un equipo Siemens de 1.5 Tesla $^{\circledR}$ (Avanto de Siemens, Erlangen, Alemania) y una bobina cardiaca. Se realizó cine eco de gradiente con sincronización cardiaca durante periodos de apnea en inspiración en reposo. Se administró adenosina a $140 \mu \mathrm{g} / \mathrm{kg} /$ minuto. En el minuto 3 de la administración de adenosina se captaron imágenes de perfusión (secuencia de primer paso ponderada en T1) administrando gadolinio, $0.1 \mathrm{mmol} / \mathrm{kg}$ de peso corporal. Las imágenes se obtuvieron en eje corto en tercio basal, medio y apical. Posterior a la adquisición las imágenes de perfusión en estrés y con la infusión de adenosina se adquirieron secuencias de cine en estrés. Entre 10 y 15 minutos después se consiguieron las imágenes de perfusión en reposo, administrando una segunda dosis de gadolinio y, finalmente 10 minutos después se adquirieron las imágenes de reforzamiento tardío.

Con el programa Argus se delimitaron los bordes endo y epicárdico (en forma semiautomática y corrección manual de ser necesario) del ventrículo izquierdo en telediástole y telesístole, con lo que se obtuvieron los parámetros de función ventricular tanto en reposo como en estrés.

Las variables volumétricas obtenidas de la RMC con adenosina fueron volumen telediastólico (VTD), VTS y FEVI de manera basal y posterior a adenosina. Se calculó la delta de la FEVI con la siguiente fórmula:

FEVI postestrés - FEVI reposo

Los responsables del cálculo de la delta de la FEVI no contaban con la información de si el paciente había tenido un resultado positivo para isquemia. Para determinar si el estudio era positivo o negativo para 
isquemia se compararon las imágenes de estrés con las de reposo. La presencia de un defecto en estrés que no se encontraba o era de menor tamaño en reposo fue consistente con isquemia miocárdica.

Se llevó a cabo análisis descriptivo para variables cuantitativas con media y desviación estándar o mediana y rango intercuartílico, frecuencias absolutas y relativas para variables cualitativas. El análisis comparativo de las variables cuantitativas se efectuó con U de Mann-Whitney o t de Student de acuerdo con la distribución y la comparación de porcentajes con chi cuadrada o prueba exacta de Fisher en frecuencias esperadas < 5. Finalmente, se realizó de curva ROC y se calcularon puntos específicos para sensibilidad y especificidad de detección de enfermedad coronaria. Se consideró estadísticamente significativo un valor de $p<0.05$. Se empleó el programa SPSS versión 22.

\section{Resultados}

Se incluyeron 59 pacientes, 41 del sexo masculino (70 \%), la media de edad fue de $59.7 \pm 10.9$ años; $37 \%$ de los estudios fueron positivos para isquemia. Las características basales se muestran en la Tabla 1. Hubo mayor porcentaje de pacientes con "EAC conocida" en el grupo de pacientes con estudios positivos en comparación con los que tuvieron estudio negativo $(p<0.001)$.

La comparación de los resultados de la RMC de los pacientes con estudio positivo versus los estudios negativos para isquemia se muestra en la Tabla 2. La FEVI basal de los pacientes con estudio positivo fue de $58.3 \%$ (48.2-65.7) versus $59 \%$ (45.3-64) de los pacientes con estudio negativo $(p=0.94)$.

La delta FEVI fue significativamente mayor en los pacientes con estudios positivos para isquemia comparada con la aquellos con estudio negativo, $-0.16 \pm$ 5.9 versus $5.3 \pm 4.7$, respectivamente $(p<0.001)$. La delta del volumen telediastólico y telesistólico fue significativamente mayor en los pacientes con estudio positivo para isquemia.

La Figura 1 muestra gráficamente la comparación de la delta FEVI entre los grupos. Ningún paciente con estudio positivo para isquemia tuvo una delta FEVI > $7.6 \%$, mientras que ningún paciente con estudio negativo tuvo una delta FEVI menor de -2.4. Se observó sobreposición de los valores entre los pacientes positivos y negativos en el rango de -2.4 y $7.6 \%$, en el que se encontraron 24 de los 37 pacientes con estudio
Tabla 1. Características basales de pacientes a quienes se les realizó resonancia magnética cardiaca con adenosina por sospecha o conocimiento de EAC

\begin{tabular}{|c|c|c|c|c|c|}
\hline & \multirow{2}{*}{\multicolumn{2}{|c|}{$\begin{array}{c}\text { Positivos } \\
(n=22)\end{array}$}} & \multicolumn{2}{|c|}{$\begin{array}{c}\text { Negativos } \\
(n=37)\end{array}$} & \multirow[t]{2}{*}{$p$} \\
\hline & & & & $\pm \mathrm{DE}$ & \\
\hline \multirow[t]{2}{*}{ Edad } & \multicolumn{2}{|c|}{$60 \pm 9.1$} & \multicolumn{2}{|c|}{$61 \pm 11.8$} & 0.740 \\
\hline & $\mathrm{n}$ & $\%$ & $\mathrm{n}$ & $\%$ & \\
\hline Diabetes mellitus & 4 & 18.2 & 10 & 27 & 0.440 \\
\hline Hipertensión & 13 & 59 & 26 & 70.2 & 0.380 \\
\hline Dislipidemia & 4 & 18.2 & 10 & 27 & 0.440 \\
\hline Tabaquismo & 13 & 59 & 14 & 37.8 & 0.113 \\
\hline EAC conocida & 14 & 64 & 6 & 16.2 & $<0.001$ \\
\hline
\end{tabular}

EAC = enfermedad arterial coronaria; se consideró que el paciente tenía enfermedad coronaria conocida si tenía el antecedente de coronariografía o angioplastia

Tabla 2. Comparación de los resultados para isquemia en pacientes con resonancia magnética cardiovascular con adenosina

\begin{tabular}{|c|c|c|c|c|c|}
\hline & \multicolumn{2}{|c|}{ Positivos $n=22$} & \multicolumn{2}{|c|}{ Negativos $n=37$} & \multirow[t]{2}{*}{$\mathbf{p}$} \\
\hline & Mediana & $\mathbf{R I}$ & Mediana & RI & \\
\hline $\begin{array}{l}\text { FEVI } \\
\text { basal }\end{array}$ & 58.3 & $48.2-65.7$ & 59 & $45.3-64$ & 0.940 \\
\hline $\begin{array}{l}\text { VTD } \\
\text { basal }\end{array}$ & 93 & 77.3-118.2 & 92.7 & $77.7-117.8$ & 0.850 \\
\hline $\begin{array}{l}\text { VTS } \\
\text { basal }\end{array}$ & 37.6 & $22.1-59.8$ & 38 & $29-52.5$ & 0.860 \\
\hline VL basal & 50.3 & $39.6-59.5$ & 54.3 & $39.8-59.9$ & 0.620 \\
\hline \multirow[t]{2}{*}{ Masa } & 76.3 & $59-116.3$ & 88 & $56.3-112.7$ & 0.730 \\
\hline & \multicolumn{2}{|c|}{ Media $\pm \mathrm{DE}$} & \multicolumn{2}{|c|}{ Media $\pm \mathrm{DE}$} & \\
\hline $\begin{array}{l}\text { Delta } \\
\text { FEVI }\end{array}$ & \multicolumn{2}{|c|}{$-0.16 \pm 5.9$} & \multicolumn{2}{|c|}{$5.3 \pm 4.7$} & $<0.001$ \\
\hline $\begin{array}{l}\text { Delta } \\
\text { VTD }\end{array}$ & \multicolumn{2}{|c|}{$15 \pm 14$} & \multicolumn{2}{|c|}{$7.1 \pm 10.4$} & 0.015 \\
\hline $\begin{array}{l}\text { Delta } \\
\text { VTS }\end{array}$ & \multicolumn{2}{|c|}{$6.3 \pm 7.7$} & \multicolumn{2}{|c|}{$-1.5 \pm 7$} & $<0.001$ \\
\hline Delta VL & \multicolumn{2}{|c|}{$8.6 \pm 10.4$} & \multicolumn{2}{|c|}{$9.3 \pm 7$} & 0.760 \\
\hline
\end{tabular}

$\mathrm{VL}=$ volumen latido, $\mathrm{VTD}=$ volumen telediastólico, $\mathrm{VTS}=$ volumen telesistólico, $\mathrm{RMC}=$ resonancia magnética cardiaca

negativo para isquemia y 15 de los 22 con estudio positivo.

La Figura 2 muestra gráficamente la comparación del volumen telesistólico entre los grupos. Ningún paciente con estudio negativo para isquemia tuvo una delta de VTS > $15 \mathrm{~mL}$, mientras que ningún paciente con estudio positivo tuvo una delta VTS menor de $-5.7 \mathrm{~mL}$. Se observó sobreposición de los valores entre los pacientes positivos y negativos en el rango 


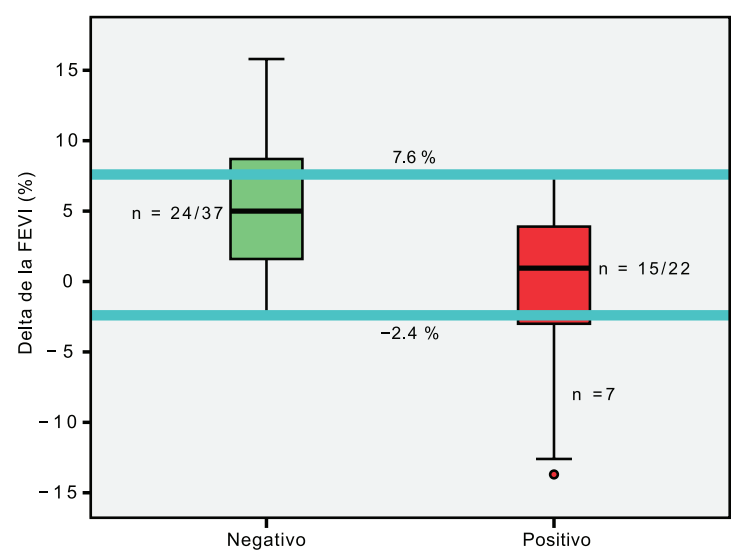

Figura 1. Comparación de delta FEVI en pacientes con estudio positivo versus negativo para isquemia. Ningún paciente con delta $F E V I>7.6 \%$ tuvo estudio positivo para isquemia miocárdica y ningún paciente con delta FEVI menor de $-2.4 \%$ tuvo estudio negativo para isquemia. FEVI = Fracción de expulsión del ventrículo izquierdo.

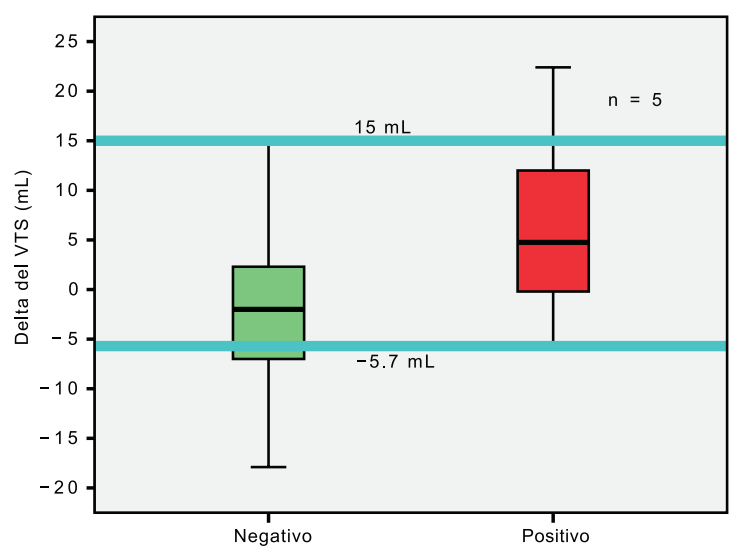

Figura 2. Comparación de volumen telesistólico en pacientes con estudio positivo versus negativo para isquemia. Ningún paciente con estudio negativo para isquemia tuvo una delta de VTS $>15 \mathrm{~mL}$. VTS = volumen telesistólico .

de 15 y $-5.7 \mathrm{~mL}$, en el cual se encontraron 17 de los 22 pacientes con estudio positivo. La Figura 3 muestra gráficamente la comparación del volumen telediastólico entre los grupos. Ningún paciente con estudio negativo para isquemia tuvo una delta de VTD $>25.5 \mathrm{~mL}$, mientras que en el rango inferior fueron muy parecidos.

En la curva ROC (Figura 4) se establecen los puntos de sensibilidad y especificidad de esta prueba según distintos puntos de corte.

\section{Discusión}

Existen diversas pruebas para el diagnóstico de enfermedad arterial coronaria, entre ellas la medicina nuclear y la resonancia magnética. Los defectos de perfusión evaluados por estos métodos son útiles para el diagnóstico y la estratificación de pacientes con cardiopatía isquémica. Desde hace tiempo, el cambio de la FEVI con estrés en el gated-SPECT ${ }^{6}$ se considera un marcador de enfermedad arterial coronaria más grave, como lo reportaron Bestetti et al., ${ }^{4,5}$ quienes también observaron que este cambio se debe al aumento del volumen telesistólico del ventrículo izquierdo.

Nuestro objetivo principal fue conocer si la delta FEVI es diferente en los pacientes con estudios positivos versus negativos para isquemia evaluados con RMC con adenosina. Los resultados fueron los esperados, ya que se encontró diferencia estadísticamente significativa en la delta FEVI por este método, además de que se confirmó que también existe diferencia significativa en la delta del VTS, como ha sido descrito en la literatura especializada. ${ }^{7}$ También encontramos diferencia significativa en el cambio del VTD. Es importante mencionar que en nuestros resultados existió sobreposición en la delta FEVI, que iba de 7.6 a -2.4\% en los estudios de los pacientes con estudio negativo versus positivo para isquemia, lo que en parte se podría explicar por la disminución de la FEVI con el uso de vasodilatadores en pacientes con estudios negativos para isquemia, como han señalado Odagiri et al. ${ }^{14}$

En nuestra opinión, este estudio es relevante ya que un valor de delta FEVI menor a $-2.4 \%$ tiene muy alta especificidad (100\%), indicativo de que pacientes con una delta FEVI igual o menor a este punto de corte tienen muy alta probabilidad de tener un estudio positivo. Por otro lado, un valor de corte de delta $\mathrm{FEVI}<0.55 \%$ tiene muy alta sensibilidad ( $86 \%$ ) para detectar estudios positivos, aunque baja especificidad. Es importante señalar que la principal limitación de nuestro estudio fue que los resultados no se comparan con el estándar de oro, la angiografía coronaria.

Si de primera instancia un estudio parece negativo para isquemia pero se encuentra una delta FEVI menor a $-2.4 \%$ debe analizarse con cautela, ya que tiene muy alta probabilidad de ser positivo; de la misma manera, un estudio que inicialmente parezca positivo, pero con una delta $\mathrm{FEVI}>0.55 \%$, es muy probable que sea negativo para isquemia.

\section{Conclusiones}

Los pacientes con estudios positivos para isquemia tienen una delta FEVI menor y mayor delta de VTS y VTD que aquellos con estudios negativos. La disminución de la FEVI posterior al estrés con adenosina en el estudio de RMC puede orientar a la necesidad de 


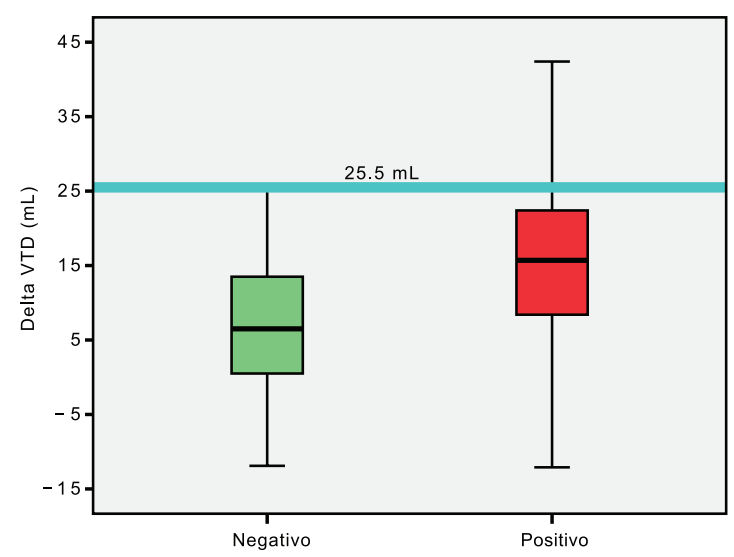

Figura 3. Comparación de la delta del VTD en pacientes con estudio positivo versus negativo para isquemia. Ningún paciente con estudio negativo para isquemia tuvo una delta de VTD $>25.5 \mathrm{~mL}$. VTD $=$ volumen telediastólico.

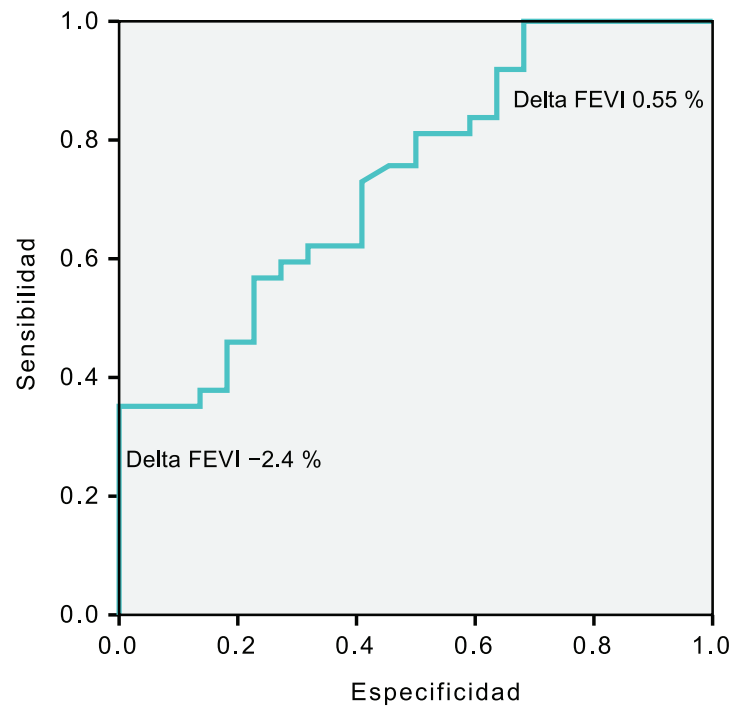

Figura 4. En la curva ROC, el valor de corte de delta FEVI de $0.55 \%$ mostró sensibilidad de $86 \%$ para la detección de estudios positivos para isquemia. El valor de corte de delta FEVI -2.4 \% tuvo una especificidad de $100 \%$ para estudios positivos para isquemia. FEVI = fracción de expulsión del ventrículo izquierdo.

una angiografía coronaria diagnóstica y eventualmente terapéutica en los pacientes con estudios anormales.
En nuestro análisis observamos sobreposición en los valores de delta FEVI en los pacientes con estudios positivos versus negativos para isquemia, por lo que es importante utilizar puntos de corte para aumentar la sensibilidad y especificidad de la prueba.

\section{Bibliografía}

1. Griffin BP, Kapadia SR, Rimmerman CM. The Cleveland Clinic Cardiology Board Review. USA: Lippincott Williams and Wilkins; 2013.

2. Instituto Nacional de Estadística, Geografía e Informática. Boletín de estadísticas vitales. México: Instituto Nacional de Estadística, Geografía e Informática; 2008.

3. Montalescot G, Sechtem U, Achenbach S, Andreotti F, Arden C, Budaj A, et al. 2013 ESC guidelines on the management of stable coronary artery disease: the Task Force on the management of stable coronary artery disease of the European Society of Cardiology. Eur Heart J. 2013:34:2949-3003.

4. Bestetti A, Triulzi A, Di-Leo C, Tagliabue L, Del-Sole A, Lomuscio A, et al. Myocardial scintigraphy by the gated SPECT method in coronary artery disease patients with post-ischemic stunning. G Ital Cardiol. 1999:29:143-148.

5. Bestetti A, Di-Leo C, Alessi A, Triulzi A, Tagliabue L, Tarolo GL. Poststress end-systolic left ventricular dilation: a marker of endocardial post-ischemic stunning. Nucl Med Commun. 2001;22:685-693.

6. Gómez-Martínez MV, Ortega-Manrique A, Álvarez-Hernando J, Theillac-Falcones B, De Jesús-Acosta M, Pereira-Del Moral R, et al. Disminución de la FEVI post-estrés en pacientes con cardiopatía isquémica y disfunción ventricular. Rev Esp Med Nucl Imagen Mol. 2014;33:160.

7. Lockie T, Ishida M, Perera D, Chiribiri A, De Silva K, Kozerke S, et al. High-resolution magnetic resonance myocardial perfusion imaging at 3.0-Tesla to detect hemodynamically significant coronary stenosis as determined by fractional flow reserve. J Am Coll Cardiol. 2011;57:70-75.

8. Pfeiffer MP, Biederman RW. Cardiac MRI: a general overview with emphasis on current use and indications. Med Clin North Am. 2015:99:849-861.

9. Selvanayagam JB, Kardos A, Francis JM, Wiesmann F, Petersen SE, Taggart DP, et al. Value of delayed-enhancement cardiovascular magnetic resonance imaging in predicting myocardial viability after surgical revascularization. Circulation. 2004:110:1535-1541.

10. Jahnke C, Nagel E, Gebker R, Kokocinski T, Kelle S, Manka R, et al. Prognostic value of cardiac magnetic resonance stress tests: adenosine stress perfusion and dobutamine stress wall motion imaging. Circulation. 2007;115:1769-1776.

11. Greenwood JP, Maredia N, Younger JF, Brown J, Nixon J, Everett C, et al. Cardiovascular magnetic resonance and single-photon emission computed tomography for diagnosis of coronary heart disease (CEMARC): a prospective trial. Lancet. 2012;379:453-460.

12. Nandalur KR, Dwamena BA, Choudhri AF, et al. Diagnostic performance of stress cardiac magnetic resonance imaging in the detection of coronary artery disease: a meta-analysis. J Am Coll Cardiol. 2007;50(14):1343-1353.

13. Schwitter J, Wacker CM, Wilke N, Al-Saadi N, Sauer E, Huettle K, et al. MR-IMPACT II: Magnetic Resonance Imaging for Myocardial Perfusion Assessment in Coronary artery disease Trial: perfusion-cardiac magnetic resonance vs. single-photon emission computed tomography for the detection of coronary artery disease: a comparative multicenter, multivendor trial. Eur Heart J. 2013;34(10):775-781.

14. Odagiri K, Machii M, Tanaka T, Uehara A, Kurata C, Sakahara H, et al. Vasodilator stress impairs the left ventricular function obtained with gated single photon emission computed tomography in patients with known or suspected coronary artery disease. Circ J. 2010;74:2666-2673. 